\title{
Galicismos en un dialecto español de Luisiana (Parroquias de Sabine y Natchitoches)
}

El dialecto hispanoamericano que se habla en las cercanías de los pueblos de Sabine Parish y en la aldea de Spanish Lake o La Laguna Española (Natchitoches Parish), en el noroeste del estado de Luisiana, ha sido desatendido hasta hace poco por los estudiosos de la dialectología hispánica ${ }^{1}$. Según indican sus apellidos, los hispano-hablantes de estas dos áreas son descendientes de soldados y colonos españoles y mejicanos del puesto fronterizo de Los Adaes, fundado en 1727 y ocupado por los españoles hasta que se retiraron de la comarca ("East Texas") en $1773^{2}$.

1 El presente artículo fue publicado en forma preliminar en Louisiana Folklife (Natchitoches), 10 (1986), pp. 21-30. Ahora sale totalmente refundido y puesto al día. Louisa Stark estudió algunos aspectos del dialecto adaeseño ya en 1980, pero, en general, su artículo fue desatendido. En 1987, John Lipski publicó un artículo crucial, basado en sus propias encuestas en las cercanías de Zwolle; en su libro sobre el dialecto isleño (1990), también trae a colación datos importantes sobre el adaeseño. Para un estudio detallado sobre la medicina popular en los poblados de Zwolle, véase Sepulvado (1977).

2 Los antepasados de los adaeseños fueron soldados y colonos del Presidio de Nuestra Señora del Pilar de Los Adaes (1723-1773). Los mismos apellidos que siguen usándose hoy en día ya constan en los registros militares y documentos eclesiásticos de Natchitoches (Mills 1973; 1980; 1982) y se destacan en la historia de la región fronteriza entre Tejas y Luisiana desde mediados hasta finales del siglo xviII (Bolton 1915; 1970). El contacto con anglo-americanos a través de los años ha deformado la pronunciación y la ortografía, pero los más de los apellidos originales siguen siendo reconocibles y, en la tradición oral, la pronunciación original sigue manteniéndose entre la gente anciana. No carece de interés en el presente contexto pasar lista de los apellidos de la comarca de Zwolle-Ebarb-Noble y de Spanish Lake: Ebarb (< Ybarbo, Ibarvo), Parrie (< Parilla), Paddie (< Padilla), Emanus (<Jiménez), Garsie, Garsee (< García), Remedies (< Ramírez), Sepulva o Sepulvado (< Sepúlveda), Manshack (<Menchaca), Malmay (< Bermejo), Basco (< Vázquez), Leone (< León), Rivers ( $<$ de los Ríos), amén de Martínez, Cortínez, Hernández, López y Sánchez. En Spanish Lake constan otros apellidos adaeseños de antiguo abolengo: Onishea (< Onís), Solice o Solees (<Solis), Ocon (< O'Conor), Moore (<Mora), Tobal (<Tobar), Bustamente o Bustamento (< Bustamante), Davis y quizás también Deers (< Díaz), Morvan, Corrales, Flores, Vernales. Al nordeste de Spanish Lake, cerca de Campti, sobrevivió el apellido Parilla en forma afrancesada: Parielle, para luego sufrir una transformación anglo-sajona: Pardee. En la comunidad de Gorum, al sureste de Los Adaes, se en- 
Basándonos en el nexo histórico entre el dialecto y el puesto de Los Adaes, hemos propuesto la denominación adaeseño ${ }^{3}$, para diferenciar el dialecto nordestino de las otras dos hablas españolas de la época colonial que aún sobreviven en Luisiana: el isleño de St. Bernard Parish y el dialecto bruli de los alrededores de Donaldsonville (Ascension, Assumption e Iberville Parishes) ${ }^{4}$. Los numerosos préstamos del nahuatl constituyen una

cuentran los apellidos Basco (< Vázquez), Vascocu, Lodrige (< Rodríguez) y Delacerda, pero el dialecto mismo ha desaparecido. Entre los criollos de Cane River, Morin se transformó en Moran, la forma francesa. Aquí también el francés reemplazó el español, para luego ceder ante el inglés. Todas estas comunidades (Campti, Gorum, Cane River) quedan al este de la frontera colonial entre Luisiana y Tejas, en un área donde el francés iba a predominar. Más al oeste, la cultura hispánica siguió en pie. En Zwolle-Ebarb-Noble, algunos apellidos franceses fueron transformados: Meshell $(<$ Michel); Ezernack (< Sarnaque). Con el tiempo, el aluvión de inmigrantes anglos que invadió Tejas hubo de anegar la cultura hispánica de la región (entre 1830-1850) y los maestros de escuela anglo-hablantes pudieron transformar a su antojo los antiguos apellidos españoles. Y sigue el proceso: ¡Un nuevo parque cerca de Zwolle, sobre el Arroyo de San Miguel, ahora se designa Sammy Gill Park! Ante tales enormidades, parece increíble la supervivencia siquiera de lo poco que aún se ha conservado. Para el contexto histórico de los poblados adaeseños, véase John (1975: 448-450 et alibi) y para estudios detallados de Los Adaes, su historia y su arqueología, Gregory y McCorkle (1980-1981) y Gregory (1983). Sobre las contribuciones amerindias a la población y la cultura de los adaeseños, véanse Opler (1983) y Kniffen, Gregory y Stokes (1987: 126, 128, 234, 303-305).

3 Los mismos adaeseños usan la palabra español (sin más) para referirse a su propio dialecto. Proyectamos un estudio detallado sobre el dialecto adaeseño. Nuestros amigos en las varias comunidades han sido de lo más gentiles al apoyar nuestro proyecto. Queremos recordar de manera especial a los llorados amigos, Sr. Martin Ebarb, Sr. Frank Martínez, Sr. Wesley Martínez y Sra., Sra. Elvira Parrie, y Sr. Ed Procell. Numerosos adaeseño-hablantes contemporáneos siguen trabajando con nosotros: En las comunidades de Zwolle-Ebarb-Noble, nos complace dar las gracias a los siguientes informantes: Henry Garcee, Maggie Manshak, Clarence Parrie, Cleve y Rosalie Parrie, Tommy Parrie, Callie Procell, y Sally Procell (de Noble) y para el subdialecto de Spanish Lake, Buddy Bustamento y Sra., Matty Corales, Mary Davis, Fred Irvan, Blas Moore y Agnes Solees Moore, Polly Morvan Hightower, Elmer Ocon, Marcelena Ocon, Randal Pleasant, Irma Sánchez, y P. H. (Pink) Solees y Sra. Nos complace expresar aquí, de todo corazón, nuestro profundo agradecimiento a todos estos amigos por la hospitalidad con que nos han recibido y por haber compartido con nosotros sus preciosos recuerdos de una lengua y una cultura que ya se aproximan al ocaso. También tenemos que dar las gracias a Mary Van Rheenan y Janet Shoemaker, cuyos conocimientos de las comunidades de Sabine Parish nos resultaron utilísimos y quienes nos acompañaron durante varias encuestas. Con la generosidad a que ya nos tiene acostumbrados, Nicholas Spitzer ha puesto a nuestro alcance varias valiosísimas cintas recogidas por él en las comunidades de Zwolle, amén de sus profundos conocimientos personales de la etnografía y el folklore de Luisiana.

4 Sobre los dialectos isleño y bruli, véanse MacCurdy $(1950 a, 1950 b, 1959)$. Entre 1975 y 1991, con el generoso apoyo del Jean Lafitte National Historical Park (U.S. 
de las características más distintivas del dialecto adaeseño, recordando sus orígenes en el suroeste de los E.E.U.U. y en Méjico, a diferencia del isle$\tilde{n} o$ y del bruli, que son dialectos de origen canario, traídos por colonizadores que llegaron al sudeste de Luisiana en $1778^{5}$.

Como hablas románicas minoritarias-en relación con el francés, la lengua dominante $\mathrm{y}$, en la época colonial (aun durante los años de la ocupación española), la lengua oficial de Luisiana-los dos dialectos del sudeste ostentan abundantes préstamos del francés local (o cadjin) ${ }^{6}$. Tanto el isleño como el bruli usan numerosos galicismos, aunque los dos dialectos los han naturalizado de manera distinta: Los isleños normalmente adaptan los préstamos cadjins a la fonología española, mientras que los brulis suelen conservar muchos rasgos fonológicos originales, de acuerdo con el carácter del dialecto, mucho más asimilado a la influencia del francés que dominaba en todas las áreas circundantes ${ }^{7}$.

La situación lingüística del adaeseño en el noroeste de Luisiana es muy diferente. Los dialectos franceses del noroeste, que remontan a la época colonial, ya apenas existen ${ }^{8}$. $\mathrm{Al}$ escuchar el dialecto adaeseño, no resulta

National Park Service, New Orleans), S.G.A. ha realizado una serie de encuestas en las dos comunidades. Algunos de los resultados se recogen en Armistead (1992a), donde también constan publicaciones anteriores. Nótense ahora además Armistead (1991a, $1991 b, 1991 c, 1992 a, 1992 b, 1993,1994 a, 1994 b)$. En 1993, Charles Holloway, en una serie de brillantes encuestas, pudo descubrir ocho nuevos informantes brulis, cuando ya dábamos por extinguido el dialecto (Armistead 1991b: 282-283). Sobre el dialecto isleño desde la perspectiva de la muerte de las lenguas, téngase en cuenta el crucial libro de John Lipski (1990).

' MacCurdy (1950a: 19-25) ofrece un breve resumen de la historia isleña. Para más detalles, véase ahora el crucial libro de Din (1988). Téngase en cuenta también el fino estudio etnográfico de Guillotte (1982: 20-33).

6 Para el empleo del francés en documentos oficiales durante la época española, sobre todo en áreas laterales y apartadas, véase Dorrance (1935: 46).

7 Véase Armistead (1991b: 282-283, 285-286, 291-293).

8 Siguen hablando francés algunos ancianos ("criollos de color") de la comunidad de Melrose, al sur de Natchitoches (compárese G. Mills 1977: 228). En la misma ciudad de Natchitoches, algunos octogenarios aún hablan su antiguo dialecto, así como algunos ancianos de Campti y en las cercanías de Black y Clear Lakes (al norte de Natchitoches). Los acadianos (cadjins), que poblaron el sur y el centro del estado, han influído relativamente poco en el noroeste de Luisiana, así que estos dialectos locales han de reflejar más bien el francés de la frontera del siglo xviII. Convendría explorar sus posibles nexos con el francés del Valle del Misisipí, según se manifestaba en las comunidades de Missouri a principios de siglo (Dorrance 1935; Carrière 1970). Compárese McDermott (1941). Igual que el dialecto español de los adaeseños, estos islotes lingüísticos franceses han quedado muy desatendidos. Apenas reconocen su existencia los estudiosos del francés en Luisiana, quienes se han ocupado exclusiva- 
nada obvia la posible presencia de algún elemento de origen francés, ni siquiera en el léxico, cuanto menos en la fonología. Si fuéramos a buscar alguna influencia alienígena en el adaeseño, el inglés ganaría por mucho al francés, no sólo en el léxico, sino también en la fonología, como, por ejemplo, al alargar los vocales tónicas y neutralizar las átonas, de acuerdo con las prácticas fonológicas del inglés. Trátase, en fin, de un dialecto rural mejicano, conservador y arcaizante, que lleva un par de siglos de relativo aislamiento, respecto a Méjico y el resto del mundo hispánico, y sujeto a la vez a la progresiva y constante influencia del inglés como la lengua dominante de la región. Con todo, a medida que hemos ido recogiendo más y más materiales léxicos, se nos ha ido descubriendo un vocabulario de préstamos franceses-muy limitado, por cierto-pero, según creemos, no desprovisto de implicaciones históricas de cierto interés, para iluminar el trasfondo cultural del dialecto. A continuación, pasamos lista de los galicismos que hemos descubierto '

1. bayuco 'río' (ing. 'bayou') (Z., S.L.): En adaeseño, esta variante hispanizada del fr.lu. bayou indica cualquier río de corriente lenta y poco agitada. Trátase de un préstamo muy antiguo, que ya consta en el mapa de Los Adaes dibujado por Joseph Urrutia en 1763 (British Museum 00306, 17662.S), donde figura el nombre del caserío Bayou Pierre como El Bayuco de las Piedras (reflejando, claro está, una fácil etimología popular). Bayuco y sus diversos avatares tienen su origen en una voz amerindia (choctaw), bayok o bayuk, pero en fr.lu. es probable que provenga de la jerga mobiliana (Mobilian jargon), especie de lingua franca amerindia practicada antaño en muchas áreas de la costa norte del Golfo de Méjico (Crawford 1978). Según nos informa Paredes (1970: 239), la voz se emplea también en Tamaulipas (nordeste de Méjico) para referirse a los arroyos desbordados. Compárese también Santamaría, quien nos depara las excepciones

mente del dialecto cadjin, y no se ha hecho el menor esfuerzo para recoger lo poco que queda. En los años 50, aún quedaban numerosos francófonos en estas áreas. Ahora convendría montar una campaña de emergencia para salvar las últimas reliquias. Sobre el dialecto cadjin, véase sobre todo el libro de Griolet (1986) y la bibliografía ahí recogida.

9 A continuación utilizamos las siguientes abreviaturas: ad. = dialecto adaeseño; br. $=$ dialecto bruli; fr.ac. $=$ francés académico; fr.am. $=$ francés americano (en general); fr.can. = francés canadiense; fr.lu. = francés de Luisiana; fr.mo. = francés de Missouri; fr.M.V. = francés del Valle del Misisipí; fr.St.Th. = francés de St. Thomas (Islas Vírgenes); ingl. = inglés; ingl.lu. = inglés de Luisiana; is. = dialecto isleño; S.L = subdialecto adaeseño de Spanish Lake; Z. = subdialecto adaeseño de las cercanías de Zwolle y aldeas vecinas. 
'zanjón, hoyanco', de acuerdo con textos del siglo xIX: “...terrenos pantanosos y algunos bayucos... El camino está cortado por bayucos... teniendo que vadear en la baja marea diversos bayucos" (1983: 125). La documentación de nuestra voz en Méjico nos comprueba indudables contactos antiguos con hispanohablantes de la costa norte del Golfo de Méjico. Sobre fr.am. bayou, véanse Read (1931: 82); Ditchy (1932: 47); Dorrance (1935: 58); McDermott (1941: 21); Friederici (1960: 84-85, 691). La presencia de la $-c$ - en ad. es notable, al confirmar la fecha temprana del préstamo. Friederici documenta ejemplos franceses de bayouc desde antes de 1744 hasta 1798, pero en todos sus ejemplos del siglo XIX ya falta la $-c$. En is. (y sin duda alguna en br. también, aunque nos falta el dato) consta bayú, que, desde luego, también se usa corrientemente en el inglés de Luisiana. En is. hay dos formas plurales: bayules y bayuses.

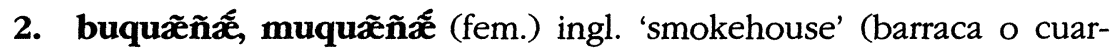
to cerrado para ahumar o acecinar carnes) (Z.): La voz ya no parece usarse corrientemente en el dialecto; hasta la fecha, sólo la hemos recogido de una única informante. La palabra de uso general es jumonera, jomonera, o a veces, por etimología popular, jamonera (pues son los jamones los que se ahuman en dicha barraca). Obviamente la forma ad. refleja el fr.am. boucanière, 'smokehouse', que se conoce en fr.lu., fr.mo. y fr.can. Véanse Read (1931: 82); Daigle (1984: 337); Ditchy (1932: 54); Dorrance (1935: 62); McDermott (1941: 31); Dionne (1974: 88); Massicotte (1978: 358); Société (1968: 137). Para la etimología de boucane 'humo', véanse Read (1931: 83) y Friederici (1960: 96-97). Is. y br. también conocen derivados de boucane y boucanière: is., br. bucana 'humo'; is. bucanera 'un nubarrón de humo'; br. bucanera 'smokehouse'.

3. camino de jiero 'ferrocarril' (Z.): Puede ser una adaptación tardía basada en fr. chemin de fer, pero conviene tener presente que camino de bierro también es de uso general en esp. y no podemos dar por definitivo que la forma ad. refleje la influencia del fr.lu. La mayoría de los préstamos del fr.lu. habrían de incorporarse al dialecto en una época muy temprana. Pero el camino de jiero no llegaría al noroeste de Luisiana sino en fecha muy tardía, reflejando, con toda seguridad, la notable evolución local de la industria maderera a finales del siglo XIX (compárese pasaportú). Muy bien puede ser, por lo tanto, que el calco semántico adaeseño se haya podido formar independientemente de su congénere español camino de hierro.

4. casquete 'hacha (pequeña)'; en concreto, la que se usa para hacer tablas de ripia: ingl. 'shingling axe' (Z., S.L.); casquete de mano 'hand 
held shingling axe' (Z.): Igual que is. y br., ad. ha adaptado el f.lu. cassetête 'hacha' (Read 1931: 23, 109), al asociarla con un homófono parcial, esp. casquete 'yelmo'. Casse-tête se conoce en fr.lu., fr.mo. y fr.can. en el sentido de 'hacha' y también de 'tomahawk' (hacha amerindia), igual que en fr.ac. Véanse Read (1931: 23); Daigle (1984: 28); Dorrance (1935: 65); McDermott (1941: 44). El fr.lu. también conoce el significado 'rompecabezas' (Daigle).

5. caữ̂n 'tortuga' (S.L.); 'tortuga de caparazón blando' (ingl. 'softshell turtle' = trionychidae) (Z.): Representa fr.lu. caouane o cauanne. Véanse Read (1931: 136-137; 1940: 49-52; 1951-1952: 232); Daigle (1984: 27). En fr.lu. alude a una variedad de especies de tortuga de agua dulce, pero sobre todo a las dos especies de tortuga mordedora (Common y Alligator Snapping Turtles = Chelydra serpentina y Macroclemys temmincki). La VOZ también existe en is. y MacCurdy (1950: 55) la recoge con ortografía francesa (caouane), al identificarla con la Alligator Snapping Turtle. En las encuestas de S.G.A., los isleños se mostraban indecisos: Para algunos, caữ̂́n era la Common Snapping Turtle, pero para la mayoría podría significar una tortuga cualquiera ("Yeah. They got'em. They got plenty of 'em out in the marsh!"). En is., la Alligator Snapping Turtle es, específicamente, la caữ́n caimanera (pero la mayoría de los isleños ya no conoce tal designación y prefiere usar el ingl.lu. loggerbead). La voz is. traduce el fr.lu. tortue caïman o tortue cocodrie, las dos voces son sinónimas al denominar esta chelydrida gigantesca, la mayor tortuga de agua dulce del mundo, que alcanza un máximo de $99.3 \mathrm{~kg}$ (Read 1940: 50; Conant 1975: 38). Nuestros informantes ad. se muestran inseguros respecto al género, mientras que para los is. es femenino. Seguramente también existe en br., pues igualmente se conoce en ingl.lu. Parece ser de origen antillano (Read 1931; Friederici 1960: 129); caguama se usa en Cuba, Puerto Rico y Méjico (Santamaría 1942; 1983; Malaret 1967).

6. chodrón (S.L.), sodrón (Z.), chorión (Z.), soriano (S.L.) 'cubo, balde'; sedrón (Z.) 'sartén': Ad. adapta el fr. chaudron. En fr.ac. significa 'calderón', pero, para fr.mo., Carrière (1970: 314) y Dorrance (1935) recogen chaudron 'seau de fer-blanc' (balde de estaño para agua o leche). El significado tiene que haber sido igual o parecido en el fr.lu. del noroeste, aunque en fr.lu. actual chaudron corresponde al ingl. cauldron y mientras baquet representa el ingl. bucket (Daigle 1984). (Tengamos en cuenta que el fr. del noroeste de Luisiana no era igual que el cadjin hablado en el sur, estando aquél más emparentado con las hablas de los antiguos núcleos franceses del Valle del Misisipí). En fr.can. constan 
chaudron, šadrõ y chaudronne en el sentido académico de 'calderón'. Véanse Dionne (1974: 139); Juneau (1977: 207, 212); Massicotte (1978: 505); Société (1968: 194) y lo mismo en el fr. M.V. según consta en una compulsa de documentos fechados entre 1673 y 1850: chaudron 'a large kettle, boiler, cauldron' (McDermott 1941: 49). En todas nuestras encuestas con los adaeseños, se manifestó de una manera impresionante el polimorfismo del dialecto. Hace por lo menos una generación —quizá más- que el dialecto adaeseño no se emplea para la comunicación cotidiana, fuera de la intimidad de la familia. Cuanto más se escucha el dialecto, más se capta la impresión de que cada familia y aún quizás cada individuo se va acordando del dialecto - a trancas y a barrancas - de un modo bastante diferente aún de sus vecinos más próximos. Es como si cada uno de los adaeseño-hablantes hubiera desarrollado, de una manera sorprendentemente diferente, su propio idiolecto distintivo. S.G.A. observó el mismo fenómeno entre los últimos portadores del dialecto bruli ${ }^{10}$. La forma chorión implica las siguientes etapas: chodrón > *hoirón > chorión (-dr- > -ir-). El mismo paso de $-d r$ - a $-i r$ - se produce en otra voz ad.: esp. yedra $>$ ad. yeira ingl. 'poison ivy' ('yedra venenosa' $=$ Rhus radicans) (Niering et al. 1979: 325-326; Brown 1980: 103) ${ }^{11}$. Soriano (S.L.) refleja el mismo proceso, pero -ón se reemplaza por otro sufijo. Junto con sedrón (Z.), soriano da fe del característico problema que experimenta el esp. con el fonema $/ \check{s} /$. La -ó- final de chodrón (etc.) no ostenta especial nasalización, aparte de la que normalmente caracteriza $V+n$ en esp. Chaudron también pasó al is., pero en otro sentido: chordón es una lata grande, con su tapa, como, por ejemplo, para guardar galletas. Se dice: un chordón de gayetas.

7. clepes ingl. 'fried bread' ('pan frito') (Z.): Trátase obviamente del fr.la. crêpes ingl. 'pancakes' ('hojuelas, tortitas') (Daigle 1984), pero son más típicos los beignets. No se documenta, que sepamos, ni en is., ni en br.

10 Semejante polimorfismo trasciende el que se encuentra normalmente en cualquier dialecto no estándard, para sugerir más bien una de las características más típicas de las lenguas moribundas. Iguales tendencias polimórficas, junto a grandes variaciones fonológicas, se encuentran en el dialecto bruli e incluso en algunos semi-hablantes isleños. Sala (1970: 41-42; 1983: 583-591) señala variaciones similares en el dialecto judeo-español de Bucarest (Rumanía), junto a significativas variaciones semánticas (polisemia). Sobre la muerte de las lenguas, véase, sobre todo, Dorian (1981) y Robins y Uhlenbeck (1991); también Dixon (1989) y Ellis (1974); y ahora, específicamente respecto al judeo-español, Harris (1994) y mi reseña (1995).

11 El dialecto bruli da fe del cambio - $d r$ - > -igr-: laigrar 'ladrar'; laigrón 'ladrón' (MacCurdy 1959: s.vv.). La transición $-d r$ - > $-g r$ - o -ir- se conoce en varios dialectos hispanoamericanos. Véase Lapesa (1981: 546-547); también Flores et al. (1969: 85); Friedemann y Patiño (1983: 108); Robe (1960: 43); Wagner (1949: 120, n. 9). 
8. cude (f.) ingl. 'front yard' ('patio exterior') (Z.): Responde al fr.ac. $\operatorname{cour}$ (f.) 'patio, corral de una casa'; lo mismo en fr.lu. cour 'yard'. Nótese también fr.St.Th. kour 'the yard beside or in front of a house' (Highfield 1979: 285). La voz $\operatorname{cur}(\mathrm{m}$.) se conoce en is., según consta en el romance tradicional de La Blancaniña: "¿De quién es aquel cabayo, / que está ensiyado en el cur?" (Armistead 1978: 49).

9. esprí trementina 'turpentine' (Z.): Huelga decir que se trata del fr. esprit, como en esprit de vin, esprit de sel, etc. La voz trementina se usa corrientemente en el español del suroeste de los EE. UU. (Opler 1983: 391).

10. flambó ingl. lu. 'fire pan' (cazuela con líquido combustible que se emplea para cazar de noche) (Z.): El dialecto ad. atribuye un sentido especializado al fr.ac. flambeau 'antorcha, vela, candelabro'. Iguales significados tiene en fr.lu. (Daigle 1984), pero nótese en fr.can. flambotter 'faire la pêche au flambeau' (Clapin 1974: 156); flamboter 'faire la pêche de nuit, dans un canot qui porte un flambeau d'écorce ou de bois résineux à son avant' (Dionne 1974: 325).

11. grega ingl. 'tea kettle' ('tetera') ( $\mathrm{Z}$.): En fr.lu. consta grègue o grégue en el sentido de 'drip coffee pot'; 'cafetière à filtre' (Daigle 1984; Ditchy 1932). Read (1931: 140-141; 1933: 369) conecta la voz con el esp. antillano greca, del mismo sentido. Se usa igualmente en Méjico, Venezuela y Colombia (Santamaría 1942; 1983; Di Filipo 1964; Malaret 1967). MacCurdy (1950) atribuye is. y br. grega a la influencia del fr.lu., como también parece ser el caso de la voz adaeseña.

12. pasaportú ingl. 'cross-cut saw' (sierra que corta a contrafibra; sierra de trozar); lo más típico es que tenga dos mangos (Z.): Adapta el fr.lu. passe-partout, que tiene el mismo sentido. Compárese Daigle passe-partout 'a logging or tree saw (often but not necessarily, with two handles)' (1984). La voz es de uso general en las Parroquias de Avoyelles y Terrebonne, así como en otras áreas del estado. En ad., el préstamo ha de remontarse a la época maderera de la Parroquia de Sabinas, a finales del siglo XIX. El fr.can. también conoce passe-partout, pero en el sentido de 'scie à chantourner' ('sierra de punto o de calador') (Dionne 1974: 486); también significa 'clef de loquet' ('llavín') (Société 1968: 499). En fr.ac., passe-partout tiene estos dos sentidos y también el de 'llave maestra'. En is. se conoce la voz passe-partout, como propia del francés cadjin y ya como una palabra incorporada al ingl.lu. Propiamente en is. se dice un sarrucho de dos hombres. 
13. romana 'vestido (de mujer)' (Z., S.L.): Refleja el fr.lu. romaine 'dress' (MacCurdy 1950). Is. y br. también usan romana en el mismo sentido. Nótese Ditchy (1932: 188): romaine 'sorte de robe de la forme d'une soutane'.

14. tiburón, tivurón ingl. 'bullfrog' ('rana mugidora') (Z., S.L.): En ad., increíblemente, esp. tiburón ha experimentado una grotesca transformación semántica como para designar la gigantesca rana tan característica de los pantanos y bayús de Luisiana (Rana catesbeiana), de una máxima largura de $20.3 \mathrm{~cm}$, la rana más grande de los E.E.U.U. (Conant 1975: 338). El cambio radical de significado ha de haberse inspirado en otra voz trisilábica del fr.lu.: ouaouaron 'bullfrog' (cuya sílaba final coincide con la de tiburón). Lejos del mar, el tiburón ya carecería de realidad para los adaeseños, sugiriendo la posibilidad de atribuir la voz a otro animal, no tan imponente como el tiburón, pero en todo caso bastante impresionante. El is. adoptó la voz del fr.lu. sin alterar: wawarón o wararón, mientras se guardó tegurón para designar el feroz pez marino. Para ouaouaron y sus orígenes amerindios, véanse Read (1931: 98-99; 1933: 367; 1940: 64); Ditchy (1932: 157, 216); Daigle (1984: 42); Dorrance (1935: 88); McDermott (1941: 110). Consta también en fr.can.: ouaouaron 'grosse grenouille verte' (Clapin 1974: 233; Dunn 1976: 131-132); 'gros crapaud' (Dionne 1974: 473); 'grenouille géante' (Juneau 1977: 16, 21; Société 1968: 485). Huelga decir que la voz ha de ser imitativa. Compárense, en la tradición angloamericana, la expresión jug o'rum o jugarum (lit. 'botijo de ron') y otras formaciones análogas que imitan el chillido del bullfrog (Behler y King 1979: 372; Cassidy y Hall 1985-1991). En ad. al bullfrog también se le llama rana sin más y consta además la traducción literal rana toro. Sobre los orígenes americanos de tiburón 'pez marino' durante los primeros años de la colonización, véanse Friederici (1960: 608-609); Corominas-Pascual (1980-1991: V, 484-486).

15. waguín 'carreta (de cuatro ruedas)' (Z., S.L.): El esp. adoptó el ingl. wagon en la forma vagón (de ferrocarril), por mediación del fr. vagon, que ha de basarse en la forma escrita de la palabra inglesa (CorominasPascual 1980-1991: V, 729). Fr.lu., fr.mo. y fr.can. adoptan la voz directamente del inglés en una forma idéntica a la del ad.: waguine, waghine y otras variantes ortográficas (Read 1931: 116; 1933: 370; Ditchy 1932: 216; Dorrance 1935: 100; Clapin 1974: 336; Dionne 1974: 669; Dunn 1976: 197; Juneau 1977: 112; Société 1968: 704). Como en ad. se suelen pronunciar los anglicismos con fonología anglo-americana, resulta probable que waguin ha de provenir del fr.lu. Por otra parte, waguín también se usa en is., 
pero en el sentido de un carruaje ligero de dos ruedas, reservándose carreta para cualquier vehículo más pesado (por ejemplo: carreta uey 'carreta de bueyes').

Por exiguo que sea nuestro pequeño repertorio de galicismos adaeseños, ayuda, según creemos, a calificar el dialecto como una modalidad del español peculiarmente luisianense en su origen y en su formación ${ }^{12}$. Las categorías semánticas que abarcan nuestros préstamos reflejan muy a las claras la naturaleza de los contactos entre hispanohablantes y franceses en la frontera de Luisiana y Tejas durante el siglo XVIII ${ }^{13}$. Casi todas nuestras voces francesas o tienen que ver con la topografía (1: bayuco) y con animales (5: caữ́n; 14 : tiburón), o bien -una mayoría- con la cultura material $(2,4,6-13,15)$. Estas comprenden la casa y sus alrededores (2: buquấñá,; 8: cude); utensilios y productos (6: chodrón; 9: espri; 11: grega); herramientas (4: casquete; 12: pasaportú); la indumentaria (13: romana); la comida (7: clepes); el transporte (3: camino de jiero; 15: waguín); y la caza (10: flambó). En bayuco, cauấn y tiburón, huelga decir que estamos ante la clásica situación, tantas veces repetida, del colonizador o inmigrante que se encuentra ante circunstancias naturales y animales extraños, ajenos al léxico europeo ${ }^{14}$. El vocabulario material, por su parte, nos evoca, con bastante exactitud, las dificultades o más bien las imposibilidades experimentadas por los hispanohablantes de Luisiana, al tratar de mantener contactos comerciales siquiera con Tejas y con Méjico,

12 Téngase en cuenta que ninguno de estos galicismos consta en el antiguo dialecto tejano, en otros aspectos muy afín al adaeseño, que aún sobrevive como vestigio del otro lado del Río Sabinas (Cherry Grove, al oeste de Chireno, St. Augustine County; El Moral, al sur de Nacogdoches). En marzo de 1984, S.G.A. realizó una serie de encuestas en estas comunidades. En este sentido, nos complace agradecer el indispensable apoyo de nuestros amigos, los profesores Francis E. Abernethy y James Corbin, de Stephen F. Austin University, en Nacogdoches (Tejas), quienes hicieron posible las encuestas en El Moral y en la misma ciudad de Nacogdoches. Nuestro llorado amigo, Claude Medford, de Natchitoches, nos proporcionó datos cruciales sobre el caserío de Cherry Grove. Sobre los colonos hispánicos de "East Texas", el artículo de Abernethy (1974) ofrece un importante punto de arranque. Nótese también Jordan (1981: 108$110,183)$.

13 Algunas de estas voces, como términos bastante comunes, se comparten coincidentemente con los otros dos dialectos (o con el isleño, o con el bruli, o bien con los dos): bayuco/bayú; buquấñó/bucanera; casquete; cauđén; cude/cur; grega; romana, waguin.

14 Compárese Armistead (1992b: 514). 
cuanto menos con España, en una sociedad fronteriza y aislada de sus raíces culturales y lingüísticas. Por otra parte, estas mismas voces confirman, de manera elocuente, la convivencia, la proximidad, el trato -fácil y constante- entre la población hispana y sus convecinos franceses, durante los primeros años de la colonia ${ }^{15}$.

\section{BIBLIOGRAFÍA}

ARBENETHY, Francis E., 1974: "The Spanish on the Moral, en F. E ABERNETHY (ed.), Folklore of Texan Cultures, Austin: Encino Press, 20-39.

ARMistead, Samuel G., y Hiram F. Gregory, 1986: "French Loan Words in the Spanish Dialect of Sabine and Natchitoches Parishes", Louisiana Folklife, 10, 21-30.

ARMistead, Samuel G., 1991a: "Catalan Children's Games in a Louisiana Spanish Community", en I. J. KATZ (ed.), Libraries, History, Diplomacy, and the Performing Arts: Essays in Honor of Carleton Sprague Smith, New York: Pendragon Press and New York Public Library, 115-121.

- 1991b: "Tres dialectos españoles de Luisiana", Lingüistica Española Actual, 13, 279-301.

- 1991c: "Vestigios de literatura oral hispánica en dos comunidades de Luisiana",en Juan PAREDES NúÑEZ (ed.), Estudios dedicados al profesor Juan Martinez Ruiz, Granada: Universidad de Granada, 49-57.

- 1992a: The Spanish Tradition in Louisiana, I: Isleño Folkliterature, Newark, Delaware: "Juan de la Cuesta".

- 1992b: "Portuguesismos en dos dialectos españoles de Luisiana," Revista de Filología Española, 72, 491-524.

- 1993: "Coplas tradicionales de los isleños de Luisiana", en Carmen Díaz AlaYón (ed.), Homenaje a la memoria de José Pérez Vidal, La Laguna, Tenerife: Cabildo Insular de La Palma, 175-183.

- 1994a: "Un topónimo guanche en Luisiana", Philologica Canariensia, [1], 39-50.

- 1994b: "Un préstamo sureslavo en el dialecto de los isleños de Luisiana", en Inés AZAR (ed.), El puente de las palabras: Homenaje a David Lagmanovich, Washington, D.C.: Organization of American States, 45-55.

- 1995: Reseña de Harris (1994), Language in Society, 24, 604-607.

BeHLER, John L., y F. WAYNE KING, 1979: The Audubon Society Field Guide to North American Reptiles and Amphibians, Nueva York: Alfred A. Knopf.

Bolton, Herbert Eugene, 1914: Athanase de Mézières and the Louisiana-Texas Frontier, 1768-1780, 2 tomos, Cleveland: Arthur H. Clark.

- 1970: Texas in the Middle Eighteenth Century: Studies in Spanish Colonial History and Administration, Austin: University of Texas Press.

BRown, Clair A., 1980: Wildflowers of Louisiana and Adjoining States, Baton Rouge: Louisiana State University Press.

15 Nótese cómo en algunas de estas voces se ha conservado, incluso, uno de los rasgos fonológicos más característicos del francés: la fuerte nasalización, como, por ejemplo, en buquẫñ̋ y cauđén. 
CARRIÈRE, Joseph Médard, 1970: Tales from the French Folk-Lore of Missouri, Nueva York: AMS.

CASSIDY, Frederic G., y Joan Houston Hall, 1985-1991: Dictionary of American Regional English, 2 tomos, Cambridge, Massachusetts: Harvard University Press.

ClAPIN, Sylva, 1974: Dictionnaire canadien-français, Quebec: Université Laval.

CONANT, Roger, 1975: A Field Guide to Reptiles and Amphibians of Eastern and Central North America, 2." ed., Boston: Houghton Mifflin.

CoROMInAS, Joan, y José A. PASCUAL, 1980-1991: Diccionario crítico etimológico castellano e bispánico, 6 tomos, Madrid: Gredos.

CRAWFORD, James M., 1978: The Mobilian Trade Language, Knoxville: University of Tennessee Press.

DaIGLE, Jules O., 1984: A Dictionary of the Cajun Language, Ann Arbor, Michigan: Edwards Brothers.

DI FILIPPO, M. Alario, 1964: Lexicón de colombianismos, Cartagena: Editora Bolívar.

DiN, Gilbert C., 1988: The Canary Islanders of Louisiana, Baton Rouge: Louisiana State University Press.

DiONNE, Narcisse-Eutrope, 1974: Le parler populaire des canadiens français, Quebec: Université Laval.

DITCHY, Jay K., 1932: Les Acadiens louisianais et leur parler, Paris: E. Droz.

DiXon, Robert M. W., 1989: Searching for Aboriginal Languages: Memoires of a Field Worker, Chicago: University of Chicago Press.

Dorian, Nancy C., 1981: Language Death: The Life Cycle of a Scottish Gaelic Dialect, Filadelfia: University of Pennsylvania Press.

DORRANCE, Ward Allison, 1935: The Survival of French in the Old District of Sainte Geneviève, Columbia, Missouri: University of Missouri (University of Missouri Studies, 10:2).

DunN, Oscar, 1976: Glossaire franco-canadien, Quebec: Université Laval.

EluIS, P. Berresford, 1974: The Cornish Language and its Literature, Londres-Boston: Routledge \& Kegan Paul.

FLÓREZ, Luis, et al., 1969: El español hablado en el Departamento del Norte de Santander: Datos y observación, Bogotá: Instituto Caro y Cuervo.

Friedemann, Nina S. de, y Carlos PatiNo Rosselli, 1983: Lengua y sociedad en el Palenque de San Basilio, Bogotá: Instituto Caro y Cuervo.

FRIEDERICI, Georg, 1960: Amerikanistisches Wörterbuch und Hilfswörterbuch für den Amerikanisten, 2. ${ }^{\mathrm{a}}$ ed., Hamburg: Cram-De Gruyter.

Gregory, Hiram F., y James MCCORKLE, 1980-1981: Los Adaes: Historical and Archaeological Background, Natchitoches: Northwest State University.

GREGORY, Hiram F., 1983: "Los Adaes: The Archaeology of an Ethnic Enclave", Geoscience and Man (Baton Rouge), 23, 53-57.

GRIOLET, Patrick, 1986: Cadjins et créoles en Louisiane: Histoire et survivance d'une francophonie, Paris: Payot.

GuillotTE, Joseph Valsin, 1982: Masters of the March: An Introduction to the Ethnography of the Isleños of Lower St. Bernard Parish, Louisiana, with an Annotated Bibliography, Nueva Orleans: University of New Orleans.

HARRIS, Tracy K., 1994: Death of a Language: The History of Judeo-Spanish, Newark: University of Delaware Press.

HighFIELD, Arnold R., 1979: The French Dialect of St. Thomas, U. S. Virgin Islands: A Descriptive Grammar with Texts and Glossary, Ann Arbor, Michigan: Karoma.

HollowAY, Charles E., 1993: The Death of a Dialect: Brule Spanish in Ascension Parish, Louisiana, tesis de Ph. D., Louisiana State University, Baton Rouge. 
JoHN, Elisabeth A. H., 1975: Storms Brewed in Other Men's Worlds: The Confrontation of Indians, Spanish, and French in the Soutbwest 1540-1795, College Station, Texas: Texas A \& M University Press.

JORDAN, Terry G., 1981: Trails to Texas: Southern Roots of Western Cattle Ranching, Lincoln: University of Nebraska Press.

JunEAU, Marcel, 1977: Problèmes de lexicologie québécoise, Quebec: Université Laval.

KNIFFEn, Fred B., Hiram F. GRegory y George A. STOKES, 1987: The Historic Indian Tribes of Louisiana from 1542 to the Present, Baton Rouge: Louisiana State University Press.

LAPESA, Rafael, 1981: Historia de la lengua española, 9. ${ }^{a}$ ed., Madrid: Gredos.

LIPSKI, John M., 1987: "El dialecto español de Río Sabinas: Vestigios del español mexicano en Luisiana y Texas", Nueva Revista de Filología Hispánica, 35, 111-128.

- 1990: The Language of the "Isleños: Vestigial Spanish in Louisiana, Baton Rouge: Louisiana State University Press.

MACCuRDY, Raymond R., 1950a: The Spanish Dialect in St. Bernard Parish, Louisiana, Albuquerque: University of New Mexico Press.

- 1950b: "Louisiana-French Loan-Words for 'Water-Fowl' in the Spanish of St. Bernard Parish, Louisiana”, en Urban T. Holmes et al. (eds.), Romance Studies presented to William Morton Dey, Chapel Hill: University of North Carolina, 137-142.

- 1959: "A Spanish Word-List of the 'Brulis' Dwellers of Louisiana", Hispania, 42, 547-554.

MALARET, Augusto, 1967: Vocabulario de Puerto Rico, Nueva York: Las Américas.

MASSICOTTE, Micheline, 1978: Le parler rural de l'île-aux-Grues (Québec): Documents lexicaux, Quebec: Université Laval.

MCDERmotT, John Francis, 1941: A Glossary of Mississippi Valley French 1673-1850, St. Louis: Washington University (Washington University Studies: Language and Literature, 12).

Mills, Elizabeth Shown, 1973: Natcbitoches 1729-1803: Abstracts of the Catbolic Cburch Registers of the French and Spanish Post of Fort St. Jean des Natchitoches in Louisiana, Nueva Orleans: Polyanthos Press.

- 1980: Natchitoches 1800-1826: Translated Abstracts of Register Number Five of the Catbolic Church Parish of St. François des Natchitoches in Louisiana, Nueva Orleans: Polyanthos Press.

- 1982: "Los Adaes: Statement of Officers and Soldiers, April 1737", Louisiana Genealogical Register: Baton Rouge, 259.

Mills, Gary B., 1977: The Forgotten People: Cane River's Creoles of Color, Baton Rouge: Louisiana State University Press.

NiERING, William A., et al., 1979: The Audubon Society Field Guide to North American Wildflowers: Eastern Region, Nueva York: Alfred A. Knopf.

OPLER, Morris E., 1983: "Apachean Culture Pattern and Its Origins", en A. ORTIZ (ed.), Handbook of North American Indians: Soutbwest, tomo 10, Washington: Smithsonian Press.

PAREDES, Américo, 1970: Folktales of Mexico, Chicago: University of Chicago Press.

READ, William A., 1931: Louisiana French, Baton Rouge: Louisiana State University Press.

- 1933: Review of Ditchy (1932), Zeitschrift für Französiscbe Sprache und Literatur, 57, 365-375.

- 1940: "A Score of Louisiana-French Words", Zeitschrift für Französische Sprache und Literatur, 63, 42-64.

- 1951-1952: Review of MacCurdy (1950), Romance Pbilology, 5, 231-232. 
RoBE, Stanley L., 1960: The Spanish of Rural Panama: Major Dialectal Features, Berkeley-Los Angeles: University of California Press.

ROBINS, Robert H., y Eugenius M. UHLENBECK (eds.), 1991: Endangered Languages, Oxford-Nueva York: Berg.

SALA, Marius, 1970: Estudios sobre el judeoespañol de Bucarest, trad. Flora Botton-Burlá, Ciudad de México: U.N.A.M.

- 1983-1987: "Algunos casos de polimorfismo en el judeo-español de Bucarest", Philologica Hispaniensia in Honorem Manuel Alvar, 4 tomos, Madrid: Gredos, I, 583-591.

SANTAMARÍA, Francisco J., 1942: Diccionario general de americanismos, 3 tomos, Ciudad de México: Pedro Robredo.

- 1983: Diccionario de mejicanismos, 4.. ed., Ciudad de México: Porrúa.

SEPulvado, Donald Lester, 1977: "Folk Curing in a Spanish Community", Louisiana Folklife Newsletter, 2:1, iii + 1-31.

SOCIÉTÉ DU PARLER FRANÇAIS AU CANADA, 1968: Glossaire du parler français au Canada, Quebec: Université Laval.

WAGNER, Max Leopold, 1949: Lingua e dialetti dell'America spagnola, Florencia: "Le Lingue Estere".

SAMUEL G. ARMistead

University of California, Davis

HIRAM F. GREGORY

Northwestern Louisiana State University

El dialecto hispanoamericano que se habla en las cercanías de varios pueblos de Sabine Parish y en la aldea de Spanish Lake o La Laguna Española (Natchitoches Parish), en el noroeste del estado de Luisiana, ha sido desatendido hasta hace poco por los estudiosos de la dialectología hispánica. Trátase de un arcaico y aislado dialecto mejicano, que está a punto de extinguirse. Tras señalar el trasfondo histórico de aquellas comunidades, que se remontan al primer tercio del siglo XVIII, se estudia el limitado repertorio de galicismos (15 en total) absorbidos por el dialecto. Las categorías semánticas permiten vislumbrar la naturaleza de los contactos entre hispanohablantes y franceses en la frontera de Luisiana y Tejas durante el siglo xviII.

The Spanish-American dialect still spoken around certain hamlets in Sabine and Natchitoches Parishes in Northwestern Louisiana has, until recently, been neglected by dialectologists. This is an archaic and isolated Mexican dialect, in danger of immediate extinction. After outlining the history of these communities, which go back to the first third of the 18th century, the limited number of French loan words (a total of 15) absorbed by the dialect are studied. The semantic categories represented give us a glimpse of the contacts between French and Spanish-speakers on the 18th-century frontier between Louisiana and Texas. 